\title{
Effects of polymer concentration and chain length on aggregation in physically associating polymer solutions
}

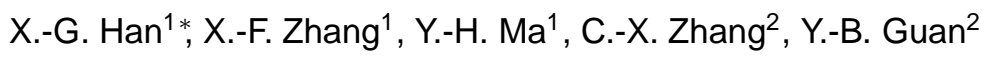 \\ ${ }^{1}$ Inner Mongolia Key Laboratory for Utilization of Bayan Obo Multi-Metallic Resources: \\ Elected State Key Laboratory, School of Mathematics, Physics and Biological engineering, \\ Inner Mongolia University of Science and Technology, 014010 Baotou, China \\ 2 Department of Physics, Jilin University, 130021 Changchun, China
}

Received July 2, 2011, in final form September 19, 2011

The effects of polymer concentration and chain length on aggregation in associative polymer solutions, are studied using self-consistent field lattice model. Only two inhomogenous morphologies, i.e. microfluctuation homogenous (MFH) and micelle morphologies, are observed in the systems with different chain lengths. The temperatures at which the above two inhomogenous morphologies first appear, which are denoted by $T_{\mathrm{MFH}}$ and $T_{\mathrm{m}}$, respectively, are dependent on polymer concentration and chain length. The variation of the logarithm of critical MFH concentration with the logarithm of chain length fulfils a linear-fitting relationship with a slope equaling -1 . Furthermore, the variation of the average volume fraction of stickers at the micellar core (AVFSM) with polymer concentration and chain length is focused in the system at $T_{\mathrm{m}}$. It is founded by calculations that the above behavior of AVFSM, is explained in terms of intrachain and interchain associations.

Key words: concentration, chain length, aggregation, associative polymer

PACS: $61.25 . \mathrm{Hp}, 87.15 . \mathrm{Nr}, 82.60 . \mathrm{Fa}$

\section{Introduction}

Physically, associating polymers are polymer chains containing a small fraction of attractive groups along the backbones. The attractive groups, for example, solvophobic groups, tend to form physical links which can play an important role in reversible junctions between different polymer chains. The junctions can be broken and recombined frequently on experimental time scales. This property of junctions makes associative polymer solutions behave reversibly when ambient conditions, such as temperature and concentrations, change. This tunable characteristic of the system produces extensive applications [1-4] that possess great potential as smart materials [5-7].

The attractive groups (also called sticker monomers) drive the self-assembly of the polymers, leading to the formation of polymeric micelles in physically associating polymer solutions (PAPSs). In telechelic [8, 9] and multiblock [10] associative polymers, flower micelles were observed. This aggregation, as well as their ability to form bridges between micelles, profoundly affect their macroscopic properties, particularly their rheological behavior, which is required in many applications. In telechelic associative polymers, the effects of architectural parameters of polymers have been assessed, such as chain length, end-group length, and chemical composition 11 15. In multiblock associative polymers, the effect of chain architecture of polymer on the property of aggregates [16, 17] was studied, which suggested that chain architecture can be an important factor in controlling macroscopic properties of the systems. However, the above studies of multiblock PAPSs were carried out in two dimensions and at low concentrations.

It is well known that self-consistent field theory (SCFT), as a mean-field theory, has been applied to the study of a great deal of problems in polymeric systems [18 21]. Recently, SCFT has

\footnotetext{
*E-mail: xghan0@163.com
} 
been applied to the study of the properties of micelles in polymer solutions $22-24]$. In the previous paper [10], given a fixed chain length, we focused on the thermodynamic properties and structure transitions in PAPSs. The microfluctuation homogenous (MFH) morphology, which corresponds to the onset of gelation [10, 25], and micelle morphology were observed. The degrees of aggregations of the above two morphologies are very different. The volume fraction of stickers in micelle morphology is much bigger than that in $\mathrm{MFH}$ morphology. In this work, the property of the aggregation in PAPAs is studied using self-consistent field lattice model. The temperatures at which MFH and micelle morphologies first appear, which are denoted by $T_{\mathrm{MFH}}$ and $T_{\mathrm{m}}$, respectively, and the average volume fraction of stickers at the micellar core, $\left\langle\phi_{\mathrm{s}}\left(r_{\mathrm{co}}\right)\right\rangle$, are calculated. Such calculations are carried out for different chain lengths and polymer concentrations. It is found that $T_{\mathrm{MFH}}, T_{\mathrm{m}}$ and $\left\langle\phi\left(r_{\mathrm{co}}\right)\right\rangle$ are dependent on chain length and polymer concentration, and the relationship of $\left\langle\phi_{\mathrm{s}}\left(r_{\mathrm{co}}\right)\right\rangle$ with chain length and polymer concentration is explained in terms of intrachain and interchain associations.

\section{Theory}

We consider a system of incompressible PAPSs, where $n_{\mathrm{P}}$ polymers, each of which is composed of $N_{\text {st }}$ segments of sticker monomer type (attractive group) and $N_{\text {ns }}$ segments of nonsticky monomer type, are distributed over a lattice. Each sticker monomer is a regularly placed apart $l$ monomer along the chain backbone. The degree of polymerization of chain is $N=N_{\mathrm{st}}+N_{\mathrm{ns}}$. In addition to polymer monomers, $n_{\mathrm{h}}$ solvent molecules are placed on the vacant lattice sites. Sticker, nonsticky monomers and solvent molecules are of the same size and each occupies one lattice site. The total number of lattice sites is $N_{\mathrm{L}}=n_{\mathrm{h}}+n_{\mathrm{P}} N$. Nearest neighbor pairs of stickers have attractive interaction $-\epsilon$ with $\epsilon>0$, which is the only non-bonded interaction in the present system. The interaction energy is expressed as:

$$
U=-\frac{\epsilon}{2} \sum_{r} \sum_{r^{\prime}} \widehat{\phi}_{\mathrm{st}}(r) \widehat{\phi}_{\mathrm{st}}\left(r^{\prime}\right)
$$

where $\sum_{r}$ means the summation over all the lattice sites $r$ and $\sum_{r^{\prime}}$ means the summation over the nearest neighbor sites of $r . \widehat{\phi}_{\text {st }}(r)=\sum_{j} \sum_{s \in \text { st }} \delta_{r, r_{j, s}}$ is the volume fraction of stickers on site $r$, where $j$ and $s$ are the indexes of chain and monomer of a polymer, respectively. $s \in$ st means that the $s$ th monomer belongs to sticker monomer type. In this simulation, however, instead of directly using the exact expression of the nearest neighbor interaction for stickers, we introduce a local concentration approximation for the non-bonded interaction [10, 26]. $\sum_{r^{\prime}} \widehat{\phi}_{\mathrm{st}}\left(r^{\prime}\right)$ in equation (2.1) is replaced with $z \widehat{\phi}_{\text {st }}(r)$, where $z$ is the coordination number of the lattice used. Within this approximation, the interaction energy is expressed as:

$$
\frac{U}{k_{\mathrm{B}} T}=-\chi \sum_{r} \widehat{\phi}_{\mathrm{st}}(r) \widehat{\phi}_{\mathrm{st}}(r),
$$

where $\chi$ is the Flory-Huggins interaction parameter in the solutions, which is equal to $\frac{z}{2 k_{\mathrm{B}} T} \epsilon$. We perform the SCFT calculations in the canonical ensemble, and the field-theoretic free energy $\mathrm{F}$ is defined as follows:

$$
\frac{F\left[\omega_{+}, \omega_{-}\right]}{k_{\mathrm{B}} T}=\sum_{r}\left\{\frac{1}{4 \chi} \omega_{-}^{2}(r)-\omega_{+}(r)\right\}-n_{\mathrm{P}} \ln Q_{\mathrm{P}}\left[\omega_{\mathrm{st}}, \omega_{\mathrm{ns}}\right]-n_{\mathrm{h}} \ln Q_{\mathrm{h}}\left[\omega_{\mathrm{h}}\right]
$$

where $Q_{\mathrm{h}}$ is the partition function of a solvent molecule subject to the field $\omega_{\mathrm{h}}(r)=\omega_{+}(r)$, which is defined as $Q_{\mathrm{h}}=\frac{1}{n_{\mathrm{h}}} \sum_{r} \exp \left[-\omega_{\mathrm{h}}(r)\right] . Q_{\mathrm{P}}$ is the partition function of a noninteraction polymer chain subject to the fields $\omega_{\text {st }}(r)=\omega_{+}(r)-\omega_{-}(r)$ and $\omega_{\text {ns }}(r)=\omega_{+}(r)$, which act on sticker and nonsticky segments, respectively. Following the scheme by Schentiens and Leermakers [27], $Q_{\mathrm{P}}$ is expressed as $Q_{\mathrm{P}}=\frac{1}{N_{\mathrm{L}}} \frac{1}{z} \sum_{r_{\mathrm{N}}} \sum_{\alpha_{\mathrm{N}}} G^{\alpha_{\mathrm{N}}}(r, N \mid 1)$, where $r_{\mathrm{N}}$ and $\alpha_{\mathrm{N}}$ denote the position and orientation of the $N$ th segment of the chain, respectively. $\sum_{r_{N}} \sum_{\alpha_{N}}$ means the summation over 
all the possible positions and orientations of the $N$ th segment of the chain. $G^{\alpha_{\mathrm{s}}}(r, s \mid 1)$ is the end segment distribution function of the sth segment of the chain, which is evaluated from the following recursive relation:

$$
G^{\alpha_{\mathrm{s}}}(r, s \mid 1)=G(r, s) \sum_{r_{s-1}^{\prime}} \sum_{\alpha_{s-1}} \lambda_{r_{\mathrm{s}}-r_{s-1}^{\prime}}^{\alpha_{\mathrm{s}}-\alpha_{s-1}} G^{\alpha_{s-1}}\left(r^{\prime}, s-1 \mid 1\right),
$$

where $G(r, s)$ is the free segment weighting factor and is expressed as

$$
G(r, s)= \begin{cases}\exp \left[-\omega_{\mathrm{st}}\left(r_{\mathrm{s}}\right)\right], & s \in \mathrm{st} \\ \exp \left[-\omega_{\mathrm{ns}}\left(r_{\mathrm{s}}\right)\right], & s \in \mathrm{ns}\end{cases}
$$

The initial condition is $G^{\alpha_{1}}(r, 1 \mid 1)=G(r, 1)$ for all the values of $\alpha_{1}$. In the above expression, the values of $\lambda_{r_{\mathrm{s}}-r_{s-1}^{\prime}}^{\alpha_{\mathrm{s}-\alpha_{s}}}$ depend on the chain model used. We assume that

$$
\lambda_{r_{\mathrm{s}}-r_{s-1}^{\prime}}^{\alpha_{s-1}-\alpha_{s-1}}= \begin{cases}0, & \alpha_{\mathrm{s}}=\alpha_{s-1} \\ 1 /(z-1), & \text { otherwise }\end{cases}
$$

This means that the chain is described as a random walk without the possibility of direct backfolding. Although self-intersections of a chain are not permitted, the excluded volume effect is sufficiently taken into account [28]. Another end segment distribution function $G^{\alpha_{s}}(r, s \mid N)$ is evaluated from the following recursive relation:

$$
G^{\alpha_{\mathrm{s}}}(r, s \mid N)=G(r, s) \sum_{r_{s+1}^{\prime}} \sum_{\alpha_{s+1}} \lambda_{r_{s+1}^{\prime}-r_{\mathrm{s}}}^{\alpha_{s+1}-\alpha_{\mathrm{s}}} G^{\alpha_{s+1}}\left(r^{\prime}, s+1 \mid N\right),
$$

with the initial condition $G^{\alpha_{\mathrm{N}}}(r, N \mid N)=G(r, N)$ for all the values of $\alpha_{\mathrm{N}}$.

Using the expressions of the end segment distribution functions, the single-segment probability distribution function $P^{(1)}(r, s)$ and the two-segment probability distribution function $P^{(2)}\left(r_{1}, s_{1} ; r_{2}, s_{2}\right)$ of the chain can be defined as follows:

$$
P^{(1)}(r, s)=\frac{1}{z N_{\mathrm{L}} Q_{\mathrm{P}}} \sum_{r_{\mathrm{s}}^{\prime}} \sum_{\alpha_{\mathrm{s}}} \frac{G^{\alpha_{\mathrm{s}}}\left(r^{\prime}, s \mid 1\right) G^{\alpha_{\mathrm{s}}}\left(r^{\prime}, s \mid N\right)}{G\left(r^{\prime}, s\right)} \delta_{r_{\mathrm{s}}^{\prime}, r},
$$

which is the normalized probability that the monomer $s$ of the chain is on the lattice site $r$;

$$
\begin{aligned}
P^{(2)}\left(r_{1}, s_{1} ; r_{2}, s_{2}\right) & =\frac{1}{z N_{\mathrm{L}} Q_{\mathrm{P}}} \sum_{r_{s_{1}}^{\prime}} \sum_{\alpha_{s_{1}}} \sum_{r_{s_{2}}^{\prime}} \sum_{\alpha_{s_{2}}} G^{\alpha_{s_{1}}}\left(r^{\prime}, s_{1} \mid 1\right) \delta_{r_{s_{1}}^{\prime}, r_{1}} \\
& \times \mathcal{G}\left(r^{\prime}, s_{1} ; r^{\prime}, s_{2}\right) G^{\alpha_{s_{2}}}\left(r^{\prime}, s_{2} \mid N\right) \delta_{r_{s_{2}}^{\prime}, r_{2}}
\end{aligned}
$$

and

$$
\mathcal{G}\left(r, s_{1} ; r, s_{2}\right)=\sum_{r_{s_{1}+1}} \sum_{\alpha_{s_{1}+1}} \ldots . . \sum_{r_{s_{2}-1}} \sum_{\alpha_{s_{2}-1}}\left\{\prod_{s=s_{1}+1}^{s_{2}-1} \lambda_{r_{\mathrm{s}}-r_{s-1}}^{\alpha_{\mathrm{s}}-\alpha_{s-1}} G(r, s)\right\} \lambda_{r_{s_{2}}-r_{s_{2}-1}}^{\alpha_{s_{2}}-\alpha_{s_{2}-1}} \quad\left(\text { for } s_{2}>s_{1}\right)
$$

yield the probability that the monomers $s_{1}$ and $s_{2}$ of the chain are on the lattice sites $r_{1}$ and $r_{2}$, respectively. It can be verified that $\sum_{r} P^{(1)}(r, s)=1$, and $\sum_{r_{2}} P^{(2)}\left(r_{1}, s_{1} ; r_{2}, s_{2}\right)=P^{(1)}\left(r_{1}, s_{1}\right)$.

Equation (2.3) can be considered as the alternative form of the self-consistent field free energy functional for incompressible polymer solutions [29]. When a local concentration approximation for the non-bonded interaction is introduced, the SCFT description of lattice model for PAPSs presented in this work is basically equivalent to that of the "Gaussian thread model" chain for the similar polymer solutions [29]. The related details are presented in [10].

Minimization of the free energy function $F$ with $\omega_{-}(r)$ and $\omega_{+}(r)$ leads to the following saddle point equations:

$$
\omega_{-}(r)=2 \chi \phi_{\text {st }}(r),
$$




$$
\phi_{\mathrm{st}}(r)+\phi_{\mathrm{ns}}(r)+\phi_{\mathrm{h}}(r)=1
$$

where

$$
\phi_{\mathrm{st}}(r)=\frac{1}{N_{\mathrm{L}}} \frac{1}{z} \frac{n_{\mathrm{P}}}{Q_{\mathrm{P}}} \sum_{s \in s t} \sum_{\alpha_{\mathrm{s}}} \frac{G^{\alpha_{\mathrm{s}}}(r, s \mid 1) G^{\alpha_{\mathrm{s}}}(r, s \mid N)}{G(r, s)}
$$

and

$$
\phi_{\mathrm{ns}}(r)=\frac{1}{N_{\mathrm{L}}} \frac{1}{z} \frac{n_{\mathrm{P}}}{Q_{\mathrm{P}}} \sum_{s \in n s} \sum_{\alpha_{\mathrm{s}}} \frac{G^{\alpha_{\mathrm{s}}}(r, s \mid 1) G^{\alpha_{\mathrm{s}}}(r, s \mid N)}{G(r, s)}
$$

are the average numbers of sticker and nonsticky segments at $r$, respectively, and $\phi_{\mathrm{h}}(r)=\frac{1}{N_{\mathrm{L}}} \frac{n_{\mathrm{h}}}{Q_{\mathrm{h}}} \exp \left[-\omega_{\mathrm{h}}(r)\right]$ is the average numbers of solvent molecules at $r$.

The saddle point is calculated using the pseudo-dynamical evolution process presented by Fredrickson et al. [30]:

$$
\begin{gathered}
\omega_{+}^{\text {new }}(r)=\omega_{+}^{\text {old }}(r)+\lambda_{+}\left(\phi_{\mathrm{st}}(r)+\phi_{\mathrm{ns}}(r)+\phi_{\mathrm{h}}(r)-1\right), \\
\omega_{-}^{\text {new }}(r)=\omega_{-}^{\text {old }}(r)+\lambda_{-}\left(\phi_{\mathrm{st}}(r)-\frac{\omega_{-}(r)}{2 \chi}\right) .
\end{gathered}
$$

The calculation is initiated from appropriately randomly-chosen fields $\omega_{+}(r)$ and $\omega_{-}(r)$, and stopped when the change of free energy $F$ between two successive iterations is reduced to the needed precision. The resulting configuration is taken as a saddle point configuration. By comparing the free energies of the saddle point configurations obtained from different initial fields, the relative stability of the observed morphologies can be assessed.

\section{Result and discussion}

In our studies, the properties of associating polymers depend on three tunable molecular parameters: $\chi$ (The Flory-Huggins interaction parameter), $N$ (Chain length) and $l$ (The number of nonsticky monomers between two neighboring stickers along the backbone, $l$ equals 9 in this paper). The calculations are performed in three-dimensional simple cubic lattice with periodic boundary condition. The aggregation behavior is first sketched using the lattice with the size $N_{\mathrm{L}}=26^{3}$. Then, the obtained results are verified using larger size lattices. The results presented below are obtained from the lattice with $N_{\mathrm{L}}=40^{3}$. Three different morphologies, i.e., the homogenous, micro-fluctuation homogenous and micelle morphologies, are observed in PAPSs. By comparing the relative stability of the observed states, the phase diagram is constructed.

Figure 1 shows the phase diagram of the systems with different chain lengths $N$. In this study, when $N$ is changed, only MFH morphology and micelles are observed as inhomogeneous morphologies. The structural morphology of $\mathrm{MFH}$ morphology does not change, and micellar shape remains spherelike (see figure 2). For $N=21$, the $\chi$ value on micellar boundary $\left(\sim 1 / T_{\mathrm{m}}\right)$ increases with decreasing $\bar{\phi}_{\mathrm{P}}$. Approaching to critical micelle concentration $\left(\bar{\phi}_{\mathrm{CMC}}=0.04\right) 1$, micellar boundary abruptly becomes steep. The $\chi$ value on $\mathrm{MFH}$ boundary $\left(\sim 1 / T_{\mathrm{MFH}}\right)$ also goes up with the decrease in $\bar{\phi}_{\mathrm{P}}$, which resembles the behavior on the micellar boundary. The critical MFH concentration $\left(\bar{\phi}_{\mathrm{CFC}}=0.37\right)$ is much higher than that of micellar morphology. Therefore, the MFH boundary intersects with the micellar boundary at $\bar{\phi}_{\mathrm{CFC}}$. When $N$ is increased, at fixed $\bar{\phi}_{\mathrm{P}}$, the $\chi$ value on micellar boundary shifts slightly to larger value, and the $\chi$ value on MFH boundary decreases markedly, which is different from that on micellar boundary. $\bar{\phi}_{\mathrm{CFC}}$ also decreases with the increase in $N$.

Figure 3 shows the variations of the logarithm of $\bar{\phi}_{\mathrm{CFC}}$ with the logarithm of $N$. It is seen that the straight line with a slope equaling -1 fits the results for all chain lengths considered in this study. The appearance of MFH morphology is considered as the onset of gelation [10, 25]. Therefore,

\footnotetext{
${ }^{1}$ Critical micelle concentration $(\mathrm{CMC})$ is generally considered as the minimum concentration of the micellar appearance. In this study, the concentration which corresponds to the $\chi$ abrupt increase on micellar boundary is regarded as CMC, which is denoted by $\bar{\phi}_{\mathrm{CMC}}$. When $N=21, \bar{\phi}_{\mathrm{CMC}}=0.04$. When $N$ is increased $\bar{\phi}_{\mathrm{CMC}}$ decreases. For $N=101$, the $\bar{\phi}_{\mathrm{CMC}}$ is not observed till $\bar{\phi}_{\mathrm{P}}=0.015$.
} 


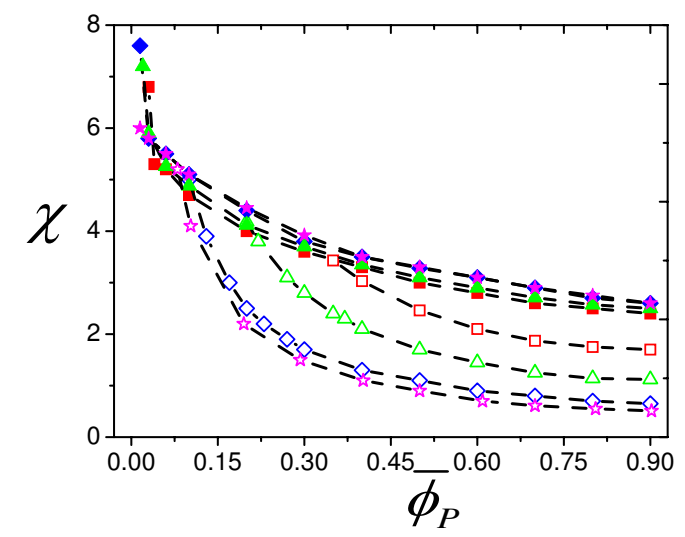

Figure 1. (Color online) The phase diagram for the systems with different chain lengths $N$. The boundaries of $\mathrm{MFH}$ and micelle morphologies are obtained. The red open and solid squares, green open and solid triangles, blue open and solid diamonds, magenta open and solid pentagons correspond to the boundaries of MFH and micelle morphologies for $N=21,41,81,101$, respectively.

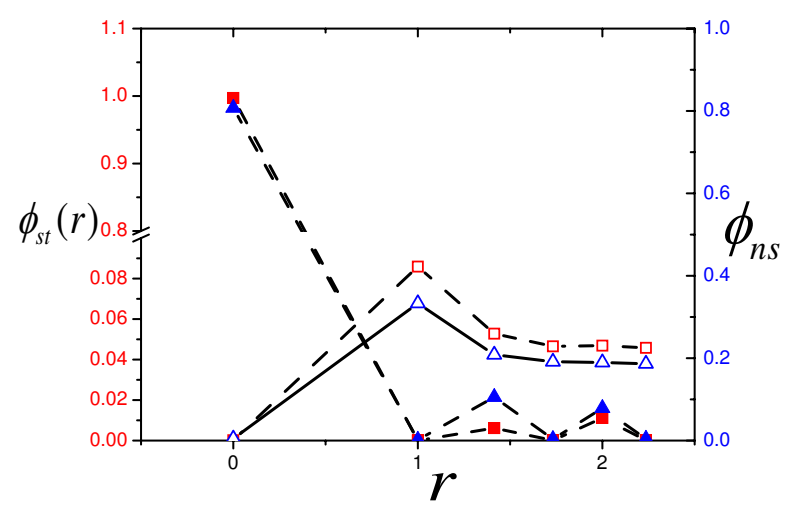

Figure 2. (Color online) The variations of the volume fractions of sticker and nonsticker components of polymers in micelle morphology with $r$ when $\bar{\phi}_{\mathrm{P}}=0.1$ and $\chi=5.5$ in the systems with $N=21$ and 101. $\mathrm{r}$ is the distance from the sticker-rich core. The red solid and open squares, blue solid and open triangles denote the volume fractions of stickers and nonstickers for $N=101$ and 21 , respectively.

critical MFH concentration $\bar{\phi}_{\mathrm{CFC}}$ should correspond to the critical gelation concentration $C_{*}$, which is analyzed in terms of the concept of chain overlap. The critical gelation concentration $C_{*}$, at which quasi-ideal coils begin to overlap, the pervaded volume of one another is related to the chain length as $N=K C_{*}^{-2}$, where $\mathrm{K}$ is a constant 31 33]. The corresponding slope of the critical concentration $C_{*}$ at quasi-ideal coil is -2 . Similarly, at the excluded volume chain, the corresponding quantity is $-5 / 4$. The slope of fitting straight line at associative polymer chain is the smallest of the above three cases. It is demonstrated that the physically associating polymer chain in solution should be elongated compared with the excluded volume chain and quasi-ideal coil.

In order to measure the degree of aggregation of stickers, an order-parameter-type variable 10] is used in this work. It is expressed as:

$$
\Phi\left(\left\{\phi_{\mathrm{st}}(r)\right\}\right)=\frac{1}{N_{\mathrm{L}}} \sum_{r}\left(\phi_{\mathrm{st}}(r)-\bar{\phi}_{\mathrm{st}}\right)^{2}=\frac{1}{N_{\mathrm{L}}} \sum_{r} \phi_{\mathrm{st}}^{2}(r)-\bar{\phi}_{\mathrm{st}}^{2},
$$




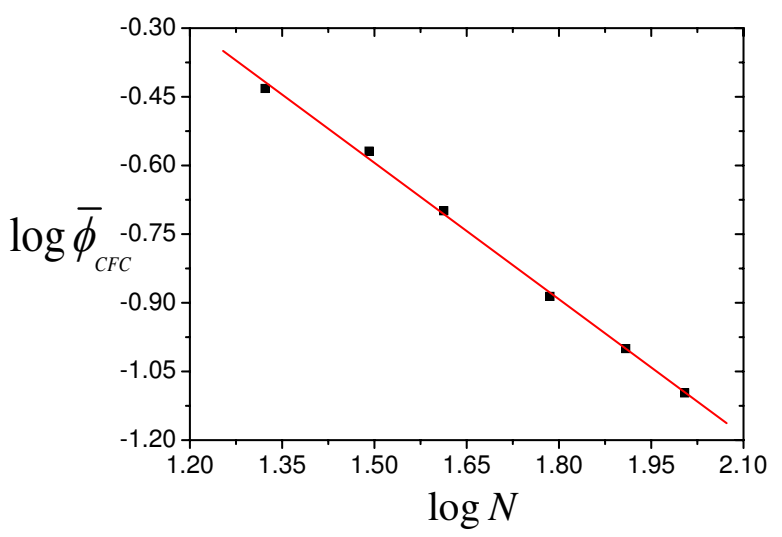

Figure 3. The logarithm of critical MFH concentration $\bar{\phi}_{\mathrm{CFC}}$ a function of the logarithm of chain length $N$, which fulfils a linear-fitting relationship with the slope equaling -1 .

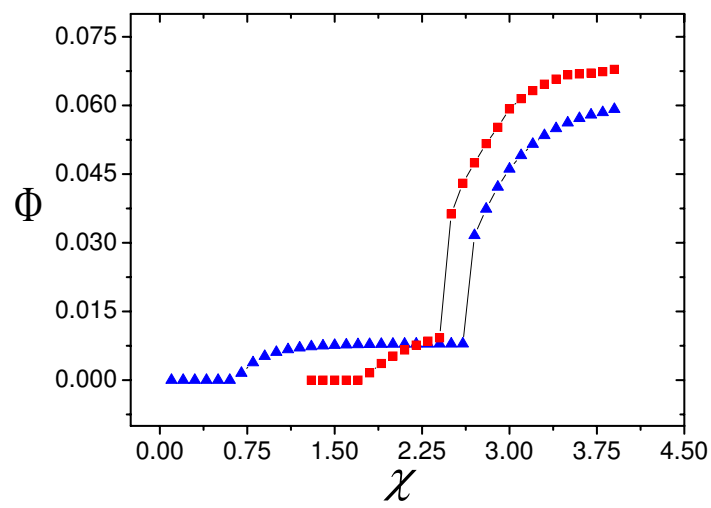

Figure 4. (Color online) The variation of the order-parameter-type variable $\Phi$ with $\chi$ near the HS-MFH and MFH-micelle transition points at $\bar{\phi}_{\mathrm{P}}=0.8$ in the systems with different chain length $N$. The red squares and blue triangles correspond to $N=21$ and $N=81$, respectively. With the increase in $\chi$, there are the appearances of two nonzero steps on the above two curves, which denote the HS-MFH and MFH-micelle transitions, respectively.

where $\Phi$ is determined by the distribution of the volume fraction of stickers $\left\{\phi_{\mathrm{st}}(r)\right\}$, which is a function of $\chi$ and $\bar{\phi}_{\mathrm{P}}$. For homogenous solutions (HS), $\Phi$ is equal to zero. Figure 4 shows the variations of $\Phi$ in MFH and micelle morphologies with an increasing $\chi$ when $\bar{\phi}_{\mathrm{P}}=0.8$ near HS$\mathrm{MFH}$ and MFH-micelle transition points, in the systems with $N=21$ and $N=81$. It is seen that the degree of the aggregation of stickers increases with an increasing $\chi$ at fixed $N$. Compared with $N=81$, although the value of $\Phi$ at fixed $\chi$ above MFH-micelle transition point at $N=21$ is larger, the variations of $\Phi$ on the above two cases with $\chi$ are similar. However, near HS-MFH transition point, the variation of $\Phi$ with $\chi$ at $N=21$ is obviously different from that of $N=81$. The value of $\Phi$ at $N=21$ increases more rapidly than that of $N=81$ when $\chi$ is increased. The heat capacity $C_{\mathrm{V}}$ is proportional to the first derivative of $\Phi$ with respect to temperature [10, 34, 35]. When $N$ is increased, the maximum of the peak of $C_{\mathrm{V}}$ decreases, and the corresponding half-width increases near the HS-MFH transition. The shape of the peak of $C_{\mathrm{V}}$ near MFH-micelle transition point does not practically change with the increase in $N$ (not shown). It is demonstrated that the change of $N$ has a greater effect on HS-MFH transition than that on MFH-micelle transition.

The aggregation number of micelles, which is used to account for some properties, for example, 


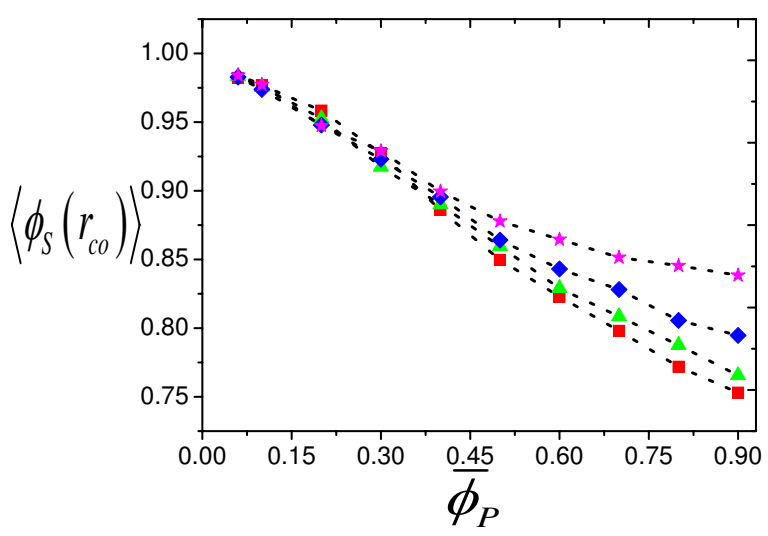

Figure 5. (Color online) The average volume fractions of stickers at micellar core as a function of $\bar{\phi}_{\mathrm{P}}$ in micellar boundary systems with different chain lengths. The red squares, green triangles, blue diamonds and magenta pentagons correspond to the boundaries of micelle morphology for $N=21,41,81,101$, respectively.

the rheological behavior of associative polymers, is a major focus in many experimental 36 38 and theoretic [39] studies. In this paper, the average volume fraction of stickers at micellar cores $\left\langle\phi_{\mathrm{s}}\left(r_{\mathrm{co}}\right)\right\rangle$ is similar to the average aggregation number of the micelle. Figure 5 shows the variations of $\left\langle\phi_{\mathrm{s}}\left(r_{\mathrm{co}}\right)\right\rangle$ on micellar boundary with $\bar{\phi}_{\mathrm{P}}$ and $N$ in the systems. Being given a fixed chain length, when $\bar{\phi}_{\mathrm{P}}$ is increased, $\left\langle\phi_{\mathrm{s}}\left(r_{\mathrm{co}}\right)\right\rangle$ decreases. When $N$ is increased, at fixed $\bar{\phi}_{\mathrm{P}}$, the variation of $\left\langle\phi_{\mathrm{s}}\left(r_{\mathrm{co}}\right)\right\rangle$ with $\bar{\phi}_{\mathrm{P}}$, does not practically change for $\bar{\phi}_{\mathrm{P}} \leqslant 0.4$, on the other hand, when $0.4<\bar{\phi}_{\mathrm{P}} \leqslant 0.9$, $\left\langle\phi_{\mathrm{s}}\left(r_{\mathrm{co}}\right)\right\rangle$ increases. The decreasing tendency of $\left\langle\phi_{\mathrm{s}}\left(r_{\mathrm{co}}\right)\right\rangle$ with $\bar{\phi}_{\mathrm{P}}$ becomes gentle with the increase in $N$ at high polymer concentrations. It is shown that the effect of the increase in $N$ on $\left\langle\phi_{\mathrm{s}}\left(r_{\text {co }}\right)\right\rangle$ is related to $\bar{\phi}_{\mathrm{P}}$.

In order to interpret the effects of $N$ and $\bar{\phi}_{\mathrm{P}}$ on the aggregation of stickers in micelle morphology, we evaluate the probabilities that a polymer chain forms intrachain and interchain associations in the system using an approach similar to the one presented in Refs. 10, 40, 41]. We suppose that there are no other sticker aggregates in the micellar system except the micellar cores because the volume fraction of stickers at micellar core approaches 1 , and that of the rest of the system is less than $10^{-1}$. A sticker in a particular chain can form an intrachain association, as well as interchain association. Ignoring the probabilities that more than two stickers of a definite chain are attached to a micellar core, the conditional probability that the sticker $s_{1}$ concerns the intrachain association, provided that the sticker $s_{1}$ is at the micelle core $r_{\mathrm{co}}$, can be expressed as:

$$
p_{\text {loop }}\left(r_{\mathrm{co}}, s_{1}\right)=\frac{1}{P^{(1)}\left(r_{\mathrm{co}}, s_{1}\right)} \sum_{s_{2} \in s t, s_{2} \neq s_{1}} P^{(2)}\left(r_{\mathrm{co}}, s_{1} ; r_{\mathrm{co}}, s_{2}\right),
$$

where $\sum_{s_{2} \in s t, s_{2} \neq s_{1}}$ means the summation over all the stickers of a polymer chain except the $s_{1}$ th one, $P^{(1)}\left(r_{\mathrm{co}}, s_{1}\right)$ and $P^{(2)}\left(r_{\mathrm{co}}, s_{1} ; r_{\mathrm{co}}, s_{2}\right)$ are the single-segment and two-segment probability distribution functions of a chain, respectively. Then, $1-p_{\text {loop }}\left(r_{\mathrm{co}}, s_{1}\right)$ is the conditional probability that the sticker $s_{1}$ is linked with those belonging to other chains when the sticker $s_{1}$ is at $r_{\mathrm{co}}$, and

$$
\mathbf{P}_{l k}\left(s_{1}\right)=\sum_{r_{\mathrm{co}}} P^{(1)}\left(r_{\mathrm{co}}, s_{1}\right) \cdot\left\lceil 1-p_{\mathrm{loop}}\left(r_{\mathrm{co}}, s_{1}\right)\right\rceil
$$

is the probability that a sticker $s_{1}$ of a chain is related to interchain association, where $\sum_{r_{c o}}$ means the summation over all the micellar cores of the system. The summation of $\mathbf{P}_{l k}\left(s_{1}\right)$ over all the stickers in a chain, $\left\langle n_{l k}\right\rangle=\sum_{s_{1}, s_{1} \in s t} \mathbf{P}_{l k}\left(s_{1}\right)$, can be viewed as the average sticker number from a particular polymer chain linked with other chains by sticker aggregates. The total number of stickers participating in interchain association in the system is expressed as $n_{\text {inte }}=$ 
$n_{\mathrm{P}}\left\langle n_{l k}\right\rangle$. The total number of stickers belonging to intrachain association is defined as $n_{\text {intr }}=$ $\left(1 / N_{\mathrm{st}}\right)\left[\sum_{s_{1}, s_{1} \in s t}\left\{\left(1 / N_{\mathrm{m}}\right)\left\{\sum_{r_{\mathrm{co}}} p_{\text {loop }}\left(r_{\mathrm{co}}, s_{1}\right)\right\}\right\}\right] \cdot\left\langle\phi_{\mathrm{s}}\left(r_{\mathrm{co}}\right)\right\rangle N_{\mathrm{m}}$, where $N_{\mathrm{m}}$ denotes the micellar number in the system.

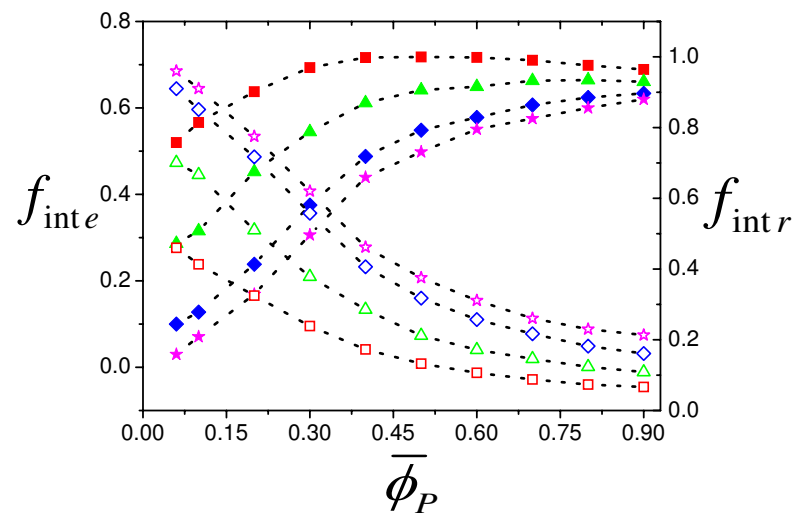

Figure 6. (Color online) The variations of the average fractions of intrachain and interchain associations at a micellar core with $\bar{\phi}_{\mathrm{P}}$ in the same systems presented in figure 5], denoted by $f_{\text {intr }}$ and $f_{\text {inte }}$, respectively. The red open and solid squares, green open and solid triangles, blue open and solid diamonds, magenta open and solid pentagons correspond to $f_{\text {intr }}$ and $f_{\text {inte }}$ for $N=21,41,81,101$, respectively.

Figure 6 shows the variations of the average fractions of intrachain and interchain associations within a micelle, denoted by $f_{\text {intr }}=n_{\text {intr }} / N_{\mathrm{m}}$ and $f_{\text {inte }}=n_{\text {inte }} / N_{\mathrm{m}}$, respectively, with $\bar{\phi}_{\mathrm{P}}$ and $N$ in the systems identical with those presented in figure 5] Being given a fixed chain length, when $\bar{\phi}_{\mathrm{P}}$ is increased, $f_{\text {intr }}$ does decrease markedly first, and then the decreasing tendency of $f_{\text {intr }}$ becomes gentle when $\bar{\phi}_{\mathrm{P}}$ exceeds some value. Except for the case of $N=21, f_{\text {inte }}$ at fixed $N$ increases markedly first, and then increases gently with the increase in $\bar{\phi}_{\mathrm{P}}$, which is contrary to $f_{\text {intr }}$. At $N=21, f_{\text {inte }}$ increases to $\bar{\phi}_{\mathrm{P}}=0.6$, then decreases slightly with the increase in $\bar{\phi}_{\mathrm{P}}$. When $N$ is increased, $f_{\text {intr }}$ at fixed $\bar{\phi}_{\mathrm{P}}$ increases, and corresponding $f_{\text {inte }}$ decreases. When $\bar{\phi}_{\mathrm{P}}$ is increased the differences of $f_{\text {intr }}$ and $f_{\text {inte }}$ in various $N$ become small. In other words, the effect of chain length on $f_{\text {intr }}$ and $f_{\text {inte }}$ at low concentrations is more pronounced than that at high concentrations.

The relative magnitude of $f_{\text {intr }}$ and $f_{\text {inte }}$ at fixed $N$ is dependent on $\bar{\phi}_{\mathrm{P}}$. Except for $N=21$, when $\bar{\phi}_{\mathrm{P}}$ is low, $f_{\text {inte }}$ at fixed $N$ is smaller than corresponding $f_{\text {intr }}$. For $N=101, f_{\text {intr }}$ equals 1 , and corresponding $f_{\text {inte }}$ equals $=0$ at $\bar{\phi}_{\mathrm{P}}=0.015$ and $\chi=6.0$. The micelle is absolutely aggregated by intrachain association. When $\bar{\phi}_{\mathrm{P}}$ exceeds some value, which is related to $N, f_{\text {inte }}$ at fixed $N$ is larger than corresponding $f_{\text {intr }}$. When $N=21, f_{\text {inte }}$ is always larger than $f_{\text {intr }}$ in the considered range of $\bar{\phi}_{\mathrm{P}}$.

In light of the variations of $\left\langle\phi_{\mathrm{S}}\left(r_{\mathrm{co}}\right)\right\rangle$ with $\bar{\phi}_{\mathrm{P}}$ and $N$ in the systems, the effect of chain length on aggregation of stickers is more pronounced at high concentrations. However, seen from respective value of $f_{\text {intr }}$ and $f_{\text {inte }}$ at fixed $\bar{\phi}_{\mathrm{P}}$, the increase of chain length is more favorable to the change of intrachain and interchain associations at low concentrations, which is different from the above conclusions drawn in light of $\left\langle\phi_{\mathrm{s}}\left(r_{\mathrm{co}}\right)\right\rangle$. It is shown that [see figure 6, being given a fixed chain length, although $f_{\text {intr }}$ is smaller than the corresponding $f_{\text {inte }}$ at high concentrations, the decrease of $\left\langle\phi_{\mathrm{S}}\left(r_{\mathrm{co}}\right)\right\rangle$ with the increase in $\bar{\phi}_{\mathrm{P}}$ is similar to that of $f_{\text {intr }}$, instead of $f_{\text {inte }}$, in the considered range of $\bar{\phi}_{\mathrm{P}}$. When $N$ is increased, $f_{\text {intr }}$ at fixed $\bar{\phi}_{\mathrm{P}}$ goes up. It is notable that the effect of chain length on $f_{\text {intr }}$ at low concentrations is much more pronounced than that at high concentrations. However, the increase of $f_{\text {intr }}$ at fixed $\bar{\phi}_{\mathrm{P}}$ does not result in the dramatical change of $\left\langle\phi_{\mathrm{s}}\left(r_{\mathrm{co}}\right)\right\rangle$ at low concentrations. At high concentrations $\left(\bar{\phi}_{\mathrm{P}}>0.4\right)$, on the contrary, $\left\langle\phi_{\mathrm{s}}\left(r_{\mathrm{co}}\right)\right\rangle$ with a big chain length has a larger value. It is reasonable that $\left\langle\phi_{\mathrm{s}}\left(r_{\mathrm{co}}\right)\right\rangle$ is also affected by $f_{\text {inte }}$. When $\bar{\phi}_{\mathrm{P}}$ is low, $f_{\text {inte }}$ at fixed $\bar{\phi}_{\mathrm{P}}$ with a long chain length is much smaller than that with a short chain. Therefore, the increase of $f_{\text {intr }}$ at fixed $\bar{\phi}_{\mathrm{P}}$ is canceled by the de- 
crease of corresponding $f_{\text {inte }}$, thus increasing in $N$. With the increase in $\bar{\phi}_{\mathrm{P}}, f_{\text {inte }}$ at fixed $\bar{\phi}_{\mathrm{P}}$ goes up markedly. The difference of $f_{\text {inte }}$ among different $N$ becomes small. The contribution of $f_{\text {intr }}$ to $\left\langle\phi_{\mathrm{s}}\left(r_{\mathrm{co}}\right)\right\rangle$ resulting from the increase in $N$ begins to appear. It is demonstrated that the effects of chain length and polymer concentration on $\left\langle\phi_{\mathrm{s}}\left(r_{\mathrm{co}}\right)\right\rangle$ do interact each other. The increase of chain length is favorable to intrachain association, and retains interchain association, which is contrary to the effect of the increase in $\bar{\phi}_{\mathrm{P}}$. When $\bar{\phi}_{\mathrm{P}}$ is increased, the effect of chain length on intrachain and interchain associations is weakened. In other words, the polymer concentration and chain length simultaneously control the formations of intrachain and interchain associations.

\section{Conclusion and summary}

Using the self-consistent field lattice model, the effects of polymer concentration $\bar{\phi}_{\mathrm{P}}$ and chain length $N$ on aggregation in physically associating polymer solutions are studied. When $N$ is changed, only two inhomogenous aggregates, i.e., the MFH and micelle morphologies, are observed in PAPSs. When $\bar{\phi}_{\mathrm{P}}$ is decreased, being given a fixed $N$, the $\chi$ values on MFH and micellar boundaries increase. At fixed $\bar{\phi}_{\mathrm{P}}$, the increase in $N$ remarkably decreases the $\chi$ value on MFH boundary, but slightly increases the $\chi$ value on micellar boundary. The logarithm of critical MFH concentration as a function of the logarithm of $N$ fulfils a fitting straight line with a slope equaling -1 , which demonstrates that the associating polymer chain in solution should be elongated compared with an excluded volume chain. Furthermore, on micellar boundary, the average volume fraction of stickers at a micellar core, $\left\langle\phi_{\mathrm{s}}\left(r_{\mathrm{co}}\right)\right\rangle$, which is similar to the average aggregation number, decreases at fixed $N$ when $\bar{\phi}_{\mathrm{P}}$ is increased. With the increase in $N$, on the other hand, $\left\langle\phi_{\mathrm{s}}\left(r_{\mathrm{co}}\right)\right\rangle$, at fixed $\bar{\phi}_{\mathrm{P}}$, does not practically change when $\bar{\phi}_{\mathrm{P}} \leqslant 0.4$. When $\bar{\phi}_{\mathrm{P}}>0.4$, on the other hand, the increase in $N$ causes the increase of $\left\langle\phi_{\mathrm{s}}\left(r_{\mathrm{co}}\right)\right\rangle$ at fixed $\bar{\phi}_{\mathrm{P}}$. There is found a decreasing tendency of variation of $\left\langle\phi_{\mathrm{s}}\left(r_{\mathrm{co}}\right)\right\rangle$ at fixed $N$, with $\bar{\phi}_{\mathrm{P}}$ is determined by intrachain association, instead of interchain association, and the magnitude of $\left\langle\phi_{\mathrm{s}}\left(r_{\mathrm{co}}\right)\right\rangle$ with different $N$ is affected by intrachain and interchain associations. At fixed $\bar{\phi}_{\mathrm{P}}$, when the difference of the contribution of interchain association to $\left\langle\phi_{\mathrm{s}}\left(r_{\mathrm{co}}\right)\right\rangle$ between different $N$ goes down to some extent, the corresponding effect of intrachain association on $\left\langle\phi_{\mathrm{s}}\left(r_{\mathrm{co}}\right)\right\rangle$ begins to appear.

The architectural parameters of polymer are important to the properties of PAPSs. In this paper, only the effect of chain length of the polymer is investigated. When the other parameters, for example, the nonsticky monomer number between two neighboring stickers $l$, are changed, the aggregation behavior is different. When $l$ is increased, the $\chi$ values on MFH and micellar boundaries rise. The increase of $l$ has a different effect on the specific heat peaks for HS-MFH and MFH-micelle transitions. Systemical studies are presented in our subsequent work. Our calculations are within the mean field framework, which may also be taken as a starting point to the further study, i.e., the direct calculation of the partition function of the system using complex Langevin simulations [29].

\section{Acknowledgements}

This research is supported by the Innovation Fund of Inner Mongolia University of Science and Technology (Grant No. 2010NC065) the High Performance Computers of Inner Mongolia University of Science and Technology.

\section{References}

1. Clark A.H., Ross-Murphy S.B., Structural and mechanical properties of biopolymer gels, In: Advances in Polymer Science, 1987, 83, 57; doi $10.1007 /$ BFb0023332

2. Slater G.W., Kist T.B.L., Ren H., Drouin G., Electrophoresis, 1998, 19, No. 10, 1525; doi:10.1002/elps.1150191003

3. Tonge S.R., Tighe B.J., Adv. Drug Delivery Rev., 2001, 53, No. 1, 109; doi:10.1016/S0169-409X(01)00223-X 
4. Taylor K.C., Nasr-El-Din H. A., J. Petrol. Sci. Eng., 1998, 19, No. 3-4, 265; doi $10.1016 /$ S0920-4105(97)00048-X.

5. Brunsveld L., Folmer B.J.B., Meijer E.W., Sijbesma R.P., Chem. Rev., 2001, 101, No. 12, 4071; doi $10.1021 / \mathrm{cr} 990125 \mathrm{q}$.

6. De Greef T. F. A., Meijer E. W., Nature, 2008, 453, No. 7192, 171; doi 10.1038/453171a

7. Cordier P., Tournilhac F., Soulié-Ziakovic C., Leibler L., Nature, 2008 451, 977; doi $10.1038 /$ nature06669.

8. Alami E., Almgren M., Brown W., Macromolecules, 1996, 29, No. 6, 2229; doi 10.1021/ma951174h

9. Chassenieux C., Nicolai T., Durand D., Macromolecules, 1997, 30, No. 17, 4952; doi $10.1021 / \mathrm{ma} 970354 \mathrm{j}$.

10. Han X.-G., Zhang C.-X., J. Chem. Phys., 2010, 132, No. 16, 164905; doi 10.1063/1.3400648

11. Chassenieux C., Nicolai T., Durand D., Macromolecules, 1998, 31, No. 12, 4035; doi $10.1021 / \mathrm{ma} 9802273$.

12. Beaudoin E., Borisov O., Lapp A., Billon L., Hiorns R.C., Franc J., Macromolecules, 2002, 35, No. 19, 7436; doi $10.1021 / \mathrm{ma} 011027 \mathrm{l}$

13. Beaudoin E., Gourierb C., Hiornsc R.C., Franc J., J. Colloid Interface Sci., 2002, 251, No. 2, 398; doi $10.1006 /$ jcis.2002.8402.

14. Lafleche F, Durand D., Nicolai T., Macromolecules, 2003, 36, No. 4, 1331; doi $10.1021 / \mathrm{ma021076d}$.

15. Lafleche F., Nicolai T., Durand D., Macromolecules, 2003, 36, No. 4, 1341; doi $10.1021 / \mathrm{ma} 0210776$

16. Balazs A.C., Gempe M.C., Lentvorski A.P., J. Chem. Phys., 1991, 95, No. 11, 8467; doi $10.1063 / 1.461276$.

17. Brown G., Chakrabarti A., J. Chem. Phys., 1992, 96, No. 10, 3251; doi $10.1063 / 1.461969$

18. Orland H., Schick M., Macromolecules, 1996, 29, No. 2, 713; doi $10.1021 / \mathrm{ma} 9508461$.

19. Matsen M.W., Schick M., Phys. Rev. Lett., 1994, 72, No. 16, 2660; doi $10.1103 /$ PhysRevLett.72.2660

20. Tang P., Qiu F., Zhang H., Yang Yu., Phys. Rev. E, 2004, 69, No. 3, 031803; doi $10.1103 /$ PhysRevE.69.031803

21. He X., Liang H., Huang L., Pan C., J. Phys. Chem. B, 2004, 108, No. 5, 1731; doi:10.1021/jp0359337

22. Cavallo A., Muller M., Binder K., Macromolecules, 2006, 39, No. 26, 9539; doi $10.1021 / \mathrm{ma061493g}$.

23. Jelinek K., Limpouchova Z., Uhlik F., Prochazka K., Macromolecules, 2007, 40, No.21, 7656; doi $10.1021 / \mathrm{ma070928c}$

24. Charlaganov M., Borisov O.V., Leermakers F.A.M., Macromolecules, 2008, 41, No. 10, 3668; doi $10.1021 / \mathrm{ma} 800130 \mathrm{q}$

25. Kumar S. K., Doughlas J. F., Phys. Rev. Lett., 2001, 87, No. 5, 188301; doi $10.1103 /$ PhysRevLett.87.188301.

26. Chen J.-Z., Zhang Ch.-X., Sun Zh.-Y., Zheng Y.-S., An L.-J., J. Chem. Phys., 2006, 124, No. 10, 104907; doi $10.1063 / 1.2176619$.

27. Leermakers F.A.M., Scheutjens J.M.H.M., J. Chem. Phys., 1988, 89, No. 5, 3264; doi $10.1063 / 1.454931$.

28. Medvedevskikh Y.G. Condens. Matter Phys., 2001, 4, No. 2, 209.

29. Fredrickson G.H., The Equilibrium Theory of Inhomogenous Polymers, Clarendon Press, Oxford, 2005; doi 10.1093/acprof:oso/9780198567295.001.0001.

30. Alexander-Katz A., Fredrickson G.H., Macromolecules, 2002, 35, 16; doi $10.1021 / \mathrm{ma011515t}$

31. Daoud M., Jannink G., J. Phys. (Paris), 1976, 37, 973; doi 10.1051/jphys:01976003707-8097300

32. Daoud M., Cotton J.P., Farnoux B., Jannink G., Sarma G., Benoit H., Duplessix C., Picot C., de Gennes P.G., Macromolecules, 1975, 8, No. 6, 804; doi 10.1021/ma60048a024

33. Klein J., Macromolecules, 1978, 11, No. 5, 852; doi 10.1021/ma60065a002.

34. Dudowicz J., Freed K. F., Douglas J. F., J. Chem. Phys., 1999, 111, No. 15, 7116; doi 10.1063/1.480004.

35. Kennedy J., Wheeler J. C., J. Chem. Phys., 1983, 78, No. 2, 953; doi 10.1063/1.444800.

36. Elliot P.T., Xing L., Wetzel W.H., Glass J.E., Macromolecules, 2003, 36, No. 22, 8449; doi $10.1021 / \mathrm{ma} 020166 \mathrm{f}$.

37. Vorobyova O., Lau W., Winnik M. A., Langmuir, 2001, 17, No. 5, 1357; doi $10.1021 /$ la0011026.

38. Serero Y., Aznar R., Porte G., Berret J.-F., Calvet D., Collet A., Viguier M., Phys. Rev. Lett., 1998, 81, No. 25, 5584; doi $10.1103 /$ PhysRevLett.81.5584

39. Meng. X.-X., Russel W. B., Macromolecules, 2005, 38, No. 2, 593; doi $10.1021 / \mathrm{ma} 048968 \mathrm{t}$

40. Matsen M. W., Thompson R. B., J. Chem. Phys., 1999, 111, No. 15, 7139; doi 10.1063/1.480006

41. Bras R. E., Shull K. R., Macromolecules, 2009, 42, No. 21, 8513; doi 10.1021/ma9013687 


\title{
Вплив концентрації полімера і довжини ланцюга на агрегацію у фізично асоційованих розчинах полімерів
}

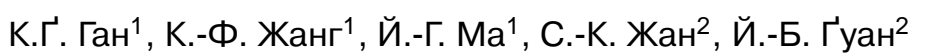 \\ 1 школа математики, фізики і біотехнологій, Університет науки і технологій Внутрішньої Монголії, \\ 014010 Баоту, Китай \\ 2 Фізичний факультет, Університет Джілін, 130021 Чанґчун, Китай
}

Використовуючи ґраткову модель самоузгодженого поля, вивчається вплив концентрації полімера і довжини ланцюга на агрегацію в асоціативних полімерних розчинах. У системах з різною довжиною ланцюга спостерігається тільки дві неоднорідні морфології, а саме мікрофлуктуаційна однорідна (MFH) і міцелярна морфології. Температури, при яких вище згадані дві морфології виникають вперше і позначаються як $T_{\mathrm{MFH}}$ і $T_{\mathrm{m}}$, відповідно, є незалежними від концентрації полімера і довжини ланцюга. Зміна логарифма критичної концентрації MFH зі зміною логарифма довжини ланцюга задовільняє співвідношенню лінійного допасування з нахилом рівним -1 . Крім того, зміна середньої об’ємної фракції стікерів при міцелярному корі (AVFSM) з концентрацією полімера і довжиною ланцюга сфокусована в системі при $T_{\mathrm{m}}$. Знайдено шляхом розрахунків, що вище згадана поведінка AVFSM пояснюється в термінах інтраланцюгових та інтерланцюгових асоціацій.

Ключові слова: концентрація, довжина ланцюга, агрегація, асоціативний полімер 


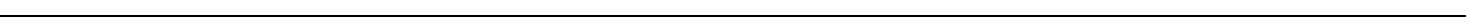

